\title{
Use of Electronic Cigarettes in Smoke-Free Spaces by Smokers: Results from the 2014-2015 Population Assessment on Tobacco and Health Study
}

\author{
Zachary R. Dunbar ${ }^{1}$, Gary Giovino ${ }^{2}$, Binnian Wei ${ }^{1}$, Richard J. O'Connor ${ }^{1}$, \\ Maciej L. Goniewicz ${ }^{1}\left(\mathbb{D}\right.$ and Mark J. Travers ${ }^{1, *}$ \\ 1 Roswell Park Comprehensive Cancer Center, Department of Health Behavior, Elm and Carlton Streets, \\ Buffalo, NY 14263, USA; Zachary.Dunbar@roswellpark.org (Z.R.D.); Binnian.Wei@roswellpark.org (B.W.); \\ Richard.OConnor@RoswellPark.org (R.J.O.C.); Maciej.Goniewicz@RoswellPark.org (M.L.G.) \\ 2 Department of Community Health and Health Behavior, School of Public Health and Health Professions, \\ University at Buffalo, The State University of New York, Buffalo, NY 14214-8028, USA; ggiovino@buffalo.edu \\ * Correspondence: Mark.Travers@roswellpark.org
}

Received: 27 November 2019; Accepted: 31 January 2020; Published: 4 February 2020

\begin{abstract}
Background: Smoke-free air policies exist to protect users and nonusers from exposure to tobacco smoke. Although electronic nicotine delivery systems (ENDS) may expose passerby to nicotine and particulate matter, few US states regulate indoor use of ENDS. The purpose of this study was to investigate reported rationales for ENDS use and reported ENDS use in public smoke-free places by dual cigarette/ENDS users. Methods: A population of ENDS/cigarette co-users $(n=2051)$ was drawn from Wave 2 of the Population Assessment of Tobacco and Health (PATH) dataset (2014-2015). Harm reduction beliefs and cessation behavior of co-users were investigated as predictors of ENDS use in public smoke-free places using logistic regression. Results: Fifty-eight percent of dual users reported past 30-day ENDS use in public smoke-free places. Reported use of ENDS to cut down on cigarette smoking (OR: 2.38, 95\% CI: 1.86, 3.05), as an alternative to quitting tobacco (OR: 1.71, 95\% CI: 1.37, 2.13), or because of belief that ENDS help people to quit cigarettes (OR: 1.52, 95\% CI: $1.20,1.92)$ were significantly associated with increased odds of ENDS use in smoke-free places. Conclusions: Beliefs that ENDS were useful as cessation tools or posed modified risk to users and nonusers were associated with elevated odds of use ENDS in locations where conventional tobacco is prohibited. Due to limitations in the survey instrument, in-home ENDS use could not be directly assessed in this analysis. However, these self-reported findings suggest that use of ENDS in public places where cigarette use is prohibited is prevalent enough to be of concern for future regulation and enforcement efforts.
\end{abstract}

Keywords: electronic cigarettes; smoke-free locations; cessation; harm reduction

\section{Introduction}

Electronic nicotine delivery system (ENDS) use in smoke-free places has been previously assessed both as a primary motivator for ENDS use [1-4] as well as a reported condition under which ENDS are commonly used [5-7]. Viewing people using ENDS in a smoke-free place may provide a mechanism through which ENDS use can become normalized in smoke-free places [8]. ENDS use in public smoke-free places is of immediate concern because aerosol emitted from indoor ENDS use introduces nicotine, tobacco-specific nitrosamines (TSNAs), fine particles, and heavy metals into the immediate environment, and although there is a wide variety of ENDS which may emit disproportionate levels of these materials, their emission in ENDS aerosol may pose a risk of passive exposure to nearby nonusers [9-14]. Further, nicotine may also be deposited on surfaces in cases where 
ENDS use occurs indoors [15], potentially leading to passive exposure to nicotine and other ENDS aerosol constituents $[16,17]$. Passive exposure to nicotine may also be associated with developmental impairments in children, such as decreased motor function, [18] lower standardized test scores, [19] and higher risk of developing learning disabilities [20]. Prolonged TSNA exposure has been associated with increased risk of lung and esophageal cancer development in animal models [21,22] and is likely a contributing factor to the increased incidence of adenocarcinoma of the lung in adults over the past two decades [23]. Similarly, fine particles have been identified as a cause of lung inflammation and irritation when inhaled in large quantities [24]. Finally, the health effects of heavy metals introduced into the indoor environment by ENDS aerosol are widely unknown [25-27]. The emission of each of these chemical species by ENDS use in homes or enclosed public places is of particular concern, as infants and small children are the population most at-risk for exposure to each of the aforementioned pollutants $[28,29]$. These deleterious health outcomes highlight the need for greater research into the frequency and motivations for use of ENDS in public smoke-free places.

This analysis examines when and why ENDS are used in order to understand the factors that may drive ENDS use in public smoke-free places. While the quantity of scientific literature on determinants of electronic nicotine delivery system (ENDS) use has grown immensely over the past five years, $[6,7,30,31]$ little is known about how potential motivators for ENDS use are related to use of these novel devices in smoke-free places. In general, ENDS use has been investigated as a potential cessation tool, with research suggesting that a primary reason for ENDS use among cigarette smokers may be to reduce or quit tobacco use $[1,2,32,33]$. Further, some research has shown that ENDS may be used to supplement or replace cigarettes in place of complete tobacco cessation $[3,4,33,34]$. Similarly, at least one study has theorized that clean indoor air laws may contribute to the selection of ENDS products whose use is harder to detect in locations with restrictions on vaping [6]. The belief that ENDS are more socially acceptable to use in public than conventional cigarettes [1,5] or the opinion that ENDS are less harmful than conventional cigarettes $[2-4,32,33,35,36]$ have also been implicated as significant predictors of general ENDS use. Each of these determinants of ENDS use may influence ENDS device preference, as the availability of flavored e-products $[1,35]$ and variety of ENDS designs [6] are each also popular motivations for ENDS use. Further, as explored in greater detail below, product characteristics may also drive safety and efficacy of a given product.

Although the FDA possesses regulatory authority over ENDS design and marketing, most clean air legislation and enforcement lies under the jurisdiction of state, regional, and local governments. In 2009, the Tobacco Control Act was passed, which gave the US Food and Drug Administration (FDA) regulatory authority over the marketing, distribution, and manufacturing of tobacco products available in the United States [37]. However, the Tobacco Control Act does not provide specific guidance on use of tobacco products in enclosed areas. Similarly, the 'deeming rule' published in August 2016 extends the regulatory authority of the FDA to include all e-cigarette devices [38]; however, the new deeming rule does not issue specific recommendations on the use of ENDS devices in indoor settings. Following the 2016 report of the Surgeon General, [29] the Centers for Disease Control and Prevention issued a call to action recommending the implementation of comprehensive clean indoor air laws that restrict the use of e-cigarettes in public, enclosed spaces; [39] however, introduction of state-level legislation regulating ENDS use in public smoke-free places has been sporadic. Comprehensive smoke-free indoor air laws (which restrict use of cigarettes in bars, restaurants, and workplaces) have been successfully implemented in twenty-five US states and territories (AZ, CA, DE, HI, IL, IA, KS, ME, MD, MA, MI, MN, MT, NE, NJ, NY, ND, OH, OR, RI, SD, UT, VT, WA, and WI, as well as Washington, DC, Puerto Rico, and the US Virgin Islands) [40]. However, despite these regulations, the use of ENDS in public smoke-free places continues to be widely unrestricted [41]. The purpose of this study was to investigate reported rationales for ENDS use and reported ENDS use in public smoke-free places by dual cigarette/ENDS users using cross-sectional data from a nationally representative cohort study in order to provide context for future tobacco control policy actions. 


\section{Materials and Methods}

\subsection{Sample}

The Population Assessment of Tobacco and Health (PATH) Study was developed and implemented by the National Institutes of Health (NIH) and FDA and is a longitudinal cohort study of tobacco use and associated health outcomes [42]. There are 28,362 individuals in the PATH Wave 2 Adult (Age 18+) public-use data file, and this population is representative of the noninstitutionalized adult population of the United States [42]. Data for Wave 2 were collected between October 2014 and October 2015. Weighting was performed on the Wave 2 Adult dataset in order to adjust for differential probability of survey selection, nonresponse, and sampling frame bias [43]. Replicate weights were employed to correct for sampling frame bias and differential rates of nonresponse. Wave 2 weights correspond to weights applied during Wave 1 (implemented in 2013-2014), correcting for nonresponse and loss to follow up in the study cohort. The second wave of the PATH study was used in this assessment, as it was the most recent wave of the PATH study that was publicly available at study onset. All percentages given below are weighted according to the PATH guidelines unless otherwise noted.

\subsection{Measures}

Current established cigarette use in this study population was defined as individuals who have smoked 100 or more cigarettes in their lifetime and self-reported current 'every day' or 'some days' current cigarette use within the previous 30 days. Current ENDS users were defined as individuals who reported ever-use of ENDS and current use on 'every day' or 'some days' within the past 30 days. Daily ENDS users were defined as individuals who self-reported 'every day' ENDS use, and nondaily ENDS users were defined as participants who reported 'some days' ENDS use within past 30 days. The definitions for 'some days' and 'every day' cigarette and ENDS use given above match the definitions used in the derived variables in the PATH dataset [44]. Individuals who were both current daily or nondaily cigarette users and current daily or nondaily ENDS users were considered 'dual' users of cigarettes and ENDS. Statistics on use frequency is provided in Table 1 below. Participants who reported smoking only cigarettes or were sole users of ENDS products were omitted from this analysis. Frequency of ENDS and tobacco use was similarly deduced from the same self-report data used to populate the overall sample of dual ENDS/tobacco users. Of the 28,362 adults in the PATH Wave 2 dataset, 2051 individuals (7.23\%) were defined as dual users. Use of ENDS in smoke-free places was defined as users who self-reported use of ENDS at times or in places (including homes and workplaces) when regular cigarettes could not be smoked at least one time over the past 30 days. This definition includes the use of ENDS in the users' own homes. The PATH survey instrument asked this question to current ENDS users regardless of their frequency of use. Disposable ENDS users were defined as individuals who reported sole use of nonrechargeable ENDS devices. Cartridge ENDS users were defined as participants that responded that the device they owned or most frequently used contains e-liquid cartridges. Similarly, tank ENDS users were defined as individuals that reported owning or most frequently used e-devices with a tank design. Individuals were also asked about beliefs associated with general ENDS use, such as the belief that ENDS may help smokers to quit using tobacco. All other variables given in Table 1 below are presented in similar format and style as what was presented to PATH respondents during the interview.

\subsection{Assessment}

Statistical assessment was performed using SAS 9.4 (SAS Institute Incorporated, Cary, NC, USA). The distribution of sociodemographic characteristics as well as selected behavioral and attitudinal norms associated with ENDS use were assessed for current dual users, stratified by use of ENDS in smoke-free places. Attitudinal and behavioral characteristics associated with ENDS use were coded as dichotomous groups. Chi-square analyses were also performed in order to assess differences in reported use of ENDS in smoke-free places by each sociodemographic, attitudinal, or behavioral 
indicator variable. Fay's method (using a coefficient of 0.3 ) was used alongside the weight replicates given in the PATH Wave 2 dataset in order to estimate the standard error of the weighting measure for each variable. Multivariable logistic regression was performed using PROC SURVEYLOGISTIC in order to assess the influence exerted by each sociodemographic, behavioral, or attitudinal predictor on odds of ENDS use in smoke-free places. Weighting factors and survey logistic procedures were utilized in accordance to the PATH Wave 2 user guidelines. Users who reported no ENDS use in smoke-free places formed the reference group for the logistic analysis. Crude and adjusted-odds ratios as well as $95 \%$ confidence intervals are given in the tables below for each predictor variable. Adjusted odds ratios correspond to beta estimates given from a multivariate logistic model containing the listed variable, with age, gender, race, ethnicity, education, and minutes to first cigarette included in the model as covariates; these data are summarized below in Table 2. Minutes to first cigarette was selected as an adjustment variable in this analysis due to its status as a proxy to indicate tobacco dependence; [45] further, minutes to first cigarette was given preference over analyst -ecoded variables such as minutes to first tobacco due to the large amount of missing data in the minutes to first ENDS variable. Minutes to first cigarette was coded as an ordinal variable, following group assignments: $0-5,5-30,31-60$, and $61+$ min. Variation inflation factors (VIF) were below 10 for each variable given in Table 2 below. All data analysis was performed using SAS 9.4 (SAS Institute Incorporated, Cary, NC, USA).

Table 1. Distribution of sociodemographic and behavioral characteristics among dual users.

\begin{tabular}{|c|c|c|c|c|c|}
\hline Variable & $n(N=2051)$ & Weighted Percent & Standard Error & $X^{2}$ & $p$-Value \\
\hline \multicolumn{6}{|l|}{ Age } \\
\hline $18-24$ & 539 & 17.97 & 0.84 & \multirow{5}{*}{18.59} & \multirow{5}{*}{$<0.001$} \\
\hline $25-34$ & 494 & 26.74 & 1.00 & & \\
\hline $35-44$ & 399 & 22.03 & 1.12 & & \\
\hline $45-55$ & 325 & 16.32 & 0.92 & & \\
\hline $55+$ & 294 & 16.93 & 1.00 & & \\
\hline \multicolumn{6}{|l|}{ Gender } \\
\hline Male & 1035 & 53.37 & 1.20 & \multirow[b]{2}{*}{6.28} & \multirow{2}{*}{0.01} \\
\hline Female & 1016 & 46.63 & 1.20 & & \\
\hline \multicolumn{6}{|l|}{ Race } \\
\hline Caucasian & 1644 & 81.92 & 0.98 & \multirow[t]{2}{*}{0.28} & \multirow[t]{2}{*}{0.60} \\
\hline Black or Other & 386 & 17.24 & 0.93 & & \\
\hline \multicolumn{6}{|l|}{ Sexual Orientation } \\
\hline LGBT+ & 208 & 9.15 & 0.69 & \multirow[t]{2}{*}{6.73} & \multirow[t]{2}{*}{0.08} \\
\hline Heterosexual & 1823 & 89.74 & 0.77 & & \\
\hline \multicolumn{6}{|l|}{ Marital Status } \\
\hline Single & 887 & 39.98 & 1.18 & \multirow{2}{*}{0.85} & \multirow{2}{*}{0.36} \\
\hline Married or other & 1164 & 60.02 & 1.18 & & \\
\hline \multicolumn{6}{|l|}{ Education Level } \\
\hline Less than High School & 300 & 13.39 & 0.74 & \multirow{4}{*}{19.18} & \multirow{4}{*}{$<0.001$} \\
\hline $\mathrm{GED}^{\wedge}$ & 243 & 11.67 & 0.82 & & \\
\hline High School Diploma & 474 & 23.95 & 1.22 & & \\
\hline Any College & 1019 & 50.28 & 1.53 & & \\
\hline \multicolumn{6}{|l|}{ Yearly Income } \\
\hline Missing & 130 & 6.60 & 0.76 & \multirow{3}{*}{1.71} & \multirow{3}{*}{0.19} \\
\hline Below $\$ 25 \mathrm{k}$ & 971 & 44.32 & 1.54 & & \\
\hline Above $\$ 25 \mathrm{~K}$ & 950 & 49.09 & 1.65 & & \\
\hline \multicolumn{6}{|l|}{ Residence Type } \\
\hline Missing & 541 & 18.12 & 0.86 & \multirow{4}{*}{0.98} & \multirow{4}{*}{0.61} \\
\hline Single family home & 987 & 53.75 & 1.38 & & \\
\hline Multi-family home or apartment & 450 & 24.33 & 1.23 & & \\
\hline Something else & 73 & 3.80 & 0.55 & & \\
\hline
\end{tabular}


Table 1. Cont

\begin{tabular}{|c|c|c|c|c|c|}
\hline Variable & $n(N=2051)$ & Weighted Percent & Standard Error & $X^{2}$ & p-Value \\
\hline \multicolumn{6}{|l|}{ Own or Rent Home } \\
\hline Own & 677 & 35.28 & 1.33 & \multirow{3}{*}{6.71} & \multirow{3}{*}{0.15} \\
\hline Rent & 1005 & 47.92 & 1.43 & & \\
\hline Something else & 351 & 15.87 & 0.91 & & \\
\hline \multicolumn{6}{|l|}{ Home Smoking Policy } \\
\hline Tobacco use prohibited at all times & 1052 & 51.90 & 1.32 & \multirow[b]{2}{*}{1.85} & \multirow{2}{*}{0.17} \\
\hline Any other policy & 994 & 47.73 & 1.32 & & \\
\hline \multicolumn{6}{|l|}{ ENDS Device Most Frequently Used } \\
\hline Cartridge or disposable user & 1171 & 58.14 & 1.41 & \multirow{2}{*}{25.48} & \multirow{2}{*}{$<0.0001$} \\
\hline Tank user & 814 & 38.43 & 1.34 & & \\
\hline \multicolumn{6}{|l|}{$\begin{array}{l}\text { Use ENDS Because: they might be less } \\
\text { harmful to people around me } \\
\text { than cigarettes }\end{array}$} \\
\hline Yes & 1621 & 79.78 & 0.90 & \multirow{2}{*}{56.04} & \multirow{2}{*}{$<0.0001$} \\
\hline No & 424 & 19.90 & 0.88 & & \\
\hline \multicolumn{6}{|l|}{$\begin{array}{l}\text { Use/used e-cigarettes because: } \\
\text { They don't smell }\end{array}$} \\
\hline Yes & 1439 & 71.38 & 1.04 & \multirow{2}{*}{43.80} & \multirow{2}{*}{$<0.0001$} \\
\hline No & 607 & 28.37 & 1.04 & & \\
\hline \multirow{2}{*}{\multicolumn{6}{|c|}{$\begin{array}{l}\text { Use/used e-cigarettes because: } \\
\text { It comes/came in flavors I like/liked }\end{array}$}} \\
\hline & & & & & \\
\hline Yes & 1425 & 67.68 & 1.12 & \multirow[b]{2}{*}{59.62} & \multirow[b]{2}{*}{$<0.0001$} \\
\hline No & 619 & 31.96 & 1.10 & & \\
\hline \multicolumn{6}{|l|}{$\begin{array}{l}\text { Using them helps people to } \\
\text { quit smoking cigarettes }\end{array}$} \\
\hline Yes & 1423 & 69.85 & 1.25 & \multirow{2}{*}{26.93} & \multirow{2}{*}{$<0.0001$} \\
\hline No & 624 & 29.95 & 1.24 & & \\
\hline $\begin{array}{c}\text { Use/used e-cigarettes as an alternative } \\
\text { quitting tobacco altogether }\end{array}$ & & & & & \\
\hline Yes & 1132 & 56.14 & 1.12 & 5383 & $<00001$ \\
\hline No & 912 & 43.53 & 1.12 & 53.83 & $<0.0001$ \\
\hline $\begin{array}{c}\text { Use/used e-cigarettes as a way of cutti } \\
\text { down on cigarette smoking }\end{array}$ & & & & & \\
\hline Yes & 1416 & 70.09 & 1.07 & 11565 & -00001 \\
\hline No & 633 & 29.85 & 1.06 & 115.65 & $<0.0001$ \\
\hline $\begin{array}{l}\text { Count of times over past } 12 \text { months, } \\
\text { stopped using tobacco for one day or } \\
\text { longer because trying to quit }\end{array}$ & & & & & \\
\hline 0 times & 1264 & 61.39 & 1.30 & & \\
\hline 1 time & 175 & 8.85 & 0.81 & & \\
\hline 2-3 times & 390 & 19.06 & 0.95 & 7.00 & 0.07 \\
\hline 4+ times & 222 & 10.70 & 0.73 & & \\
\hline Minutes after waking to first cigarett & & & & & \\
\hline less than $5 \mathrm{~min}$ & 465 & 22.99 & 1.08 & & \\
\hline $5-30 \mathrm{~min}$ & 746 & 35.42 & 1.20 & & \\
\hline $31-60 \mathrm{~min}$ & 385 & 19.26 & 1.00 & 1.54 & 0.67 \\
\hline $61+\min$ & 443 & 21.69 & 1.21 & & \\
\hline Minutes after waking to first puff on EN & & & & & \\
\hline Missing & 966 & 47.15 & 1.30 & 3.81 & 0.28 \\
\hline Less than $5 \mathrm{~min}$ & 209 & 10.05 & 0.80 & & \\
\hline 5-30 $\mathrm{min}$ & 270 & 13.24 & 0.87 & & \\
\hline $31-60 \mathrm{~min}$ & 201 & 9.52 & 0.63 & & \\
\hline $61+\min$ & 405 & 20.04 & 0.91 & & \\
\hline Tobacco/ENDS Use & & & & & \\
\hline Nondaily (Both) & 353 & 17.43 & 0.97 & & \\
\hline Nondaily Cigarette, Daily ENDS & 198 & 9.67 & 0.74 & & \\
\hline Daily (Both) & 183 & 9.46 & 0.66 & 83.25 & $<0.0001$ \\
\hline Daily Cigarette, Nondaily ENDS & 1317 & 63.44 & 1.32 & & \\
\hline
\end{tabular}


Table 1. Cont.

\begin{tabular}{|c|c|c|c|c|c|}
\hline Variable & $n(N=2051)$ & Weighted Percent & Standard Error & $X^{2}$ & $p$-Value \\
\hline \multicolumn{6}{|c|}{$\begin{array}{l}\text { Frequency of Daily ENDS Use } \\
\text { (Regardless of Tobacco Use Frequency) }\end{array}$} \\
\hline Nondaily ENDS & 1670 & 80.87 & 0.96 & \multirow{2}{*}{82.84} & \multirow{2}{*}{$<0.0001$} \\
\hline Daily ENDS & 381 & 19.13 & 0.96 & & \\
\hline \multicolumn{6}{|c|}{$\begin{array}{l}\text { Frequency of Daily Cigarette Use } \\
\text { (Regardless of ENDS Use Frequency) }\end{array}$} \\
\hline Nondaily Cigarette User & 551 & 27.1 & 1.24 & \multirow{2}{*}{5.19} & \multirow{2}{*}{0.02} \\
\hline Daily Cigarette User & 1500 & 72.9 & 1.24 & & \\
\hline
\end{tabular}

* Missing values less than $5 \%$ of total population were excluded from Table 1 above. ^ General Education Diploma. In the United States, this corresponds to certification of academic skillsets equivalent to high school degree completion. 
Table 2. Logistic model predicting odds of past 30-Day ENDS use in smoke-free locations-main effects.

\begin{tabular}{|c|c|c|c|c|c|c|c|c|c|c|c|c|}
\hline \multirow[t]{2}{*}{ Variable } & \multicolumn{3}{|c|}{$\begin{array}{l}\text { Do Not Report Using ENDS } \\
\text { in Smoke-Free Places }\end{array}$} & \multicolumn{3}{|c|}{$\begin{array}{l}\text { Report Using ENDS } \\
\text { in Smoke-Free Places }\end{array}$} & \multicolumn{3}{|c|}{ Crude Model * } & \multicolumn{3}{|c|}{ Adjusted Model ** } \\
\hline & \multicolumn{3}{|c|}{$n=862$} & \multicolumn{3}{|c|}{$n=1189$} & \multirow[b]{2}{*}{ Crude OR } & \multirow[b]{2}{*}{$95 \%$ CI LB } & \multirow[b]{2}{*}{$95 \%$ CI UB } & \multirow[b]{2}{*}{ Adj OR * } & \multirow[b]{2}{*}{$95 \%$ CI LB } & \multirow[b]{2}{*}{$95 \% \mathrm{CI} \mathrm{UB}$} \\
\hline$N=2051$ & $n$ & $\%$ & SE & $n$ & $\%$ & SE & & & & & & \\
\hline \multicolumn{13}{|c|}{ Sociodemographic Characteristics } \\
\hline \multicolumn{13}{|l|}{ Age } \\
\hline $18-24$ & 248 & 19.56 & 1.51 & 291 & 16.80 & 1.06 & 1 & - & - & 1 & - & - \\
\hline $25-34$ & 196 & 26.09 & 1.52 & 298 & 27.22 & 1.26 & 1.19 & 0.87 & 1.63 & 1.10 & 0.80 & 1.53 \\
\hline $35-44$ & 150 & 20.09 & 1.54 & 249 & 23.46 & 1.52 & 1.33 & 0.96 & 1.84 & 1.21 & 0.85 & 1.72 \\
\hline $45-55$ & 121 & 14.43 & 1.05 & 204 & 17.72 & 1.24 & 1.49 & 1.06 & 2.10 & 1.33 & 0.94 & 1.89 \\
\hline $55+$ & 147 & 19.82 & 1.61 & 147 & 14.79 & 1.23 & 0.77 & 0.55 & 1.10 & 0.70 & 0.49 & 1.00 \\
\hline \multicolumn{13}{|l|}{ Gender } \\
\hline Male & 463 & 56.87 & 1.82 & 572 & 50.79 & 1.68 & $0.75 *$ & $0.61 *$ & $0.91 *$ & $0.76 *$ & $0.62 *$ & $0.93 *$ \\
\hline Female & 399 & 43.13 & 1.82 & 617 & 49.21 & 1.68 & 1 & - & - & 1 & - & - \\
\hline \multicolumn{13}{|l|}{ Race } \\
\hline White alone & 687 & 80.20 & 1.60 & 957 & 83.18 & 1.34 & 1 & - & - & 1 & - & - \\
\hline Black or other alone & 167 & 18.97 & 1.60 & 219 & 15.96 & 1.29 & 0.79 & 0.57 & 1.09 & 0.77 & 0.55 & 1.07 \\
\hline \multicolumn{13}{|l|}{ Sexual Orientation } \\
\hline LGBT + & 75 & 7.48 & 0.97 & 133 & 10.38 & 0.95 & $1.49 *$ & $1.01 *$ & $2.20 *$ & 1.37 & 0.93 & 2.02 \\
\hline Heterosexual & 779 & 91.53 & 1.04 & 1044 & 88.42 & 1.05 & 1 & - & - & 1 & - & - \\
\hline \multicolumn{13}{|l|}{ Marital Status } \\
\hline Single & 383 & 41.22 & 1.88 & 504 & 39.07 & 1.63 & 1 & - & - & 1 & - & - \\
\hline Married or other & 479 & 58.78 & 1.88 & 685 & 60.93 & 1.63 & 1.04 & 0.83 & 1.31 & 1.03 & 0.77 & 1.38 \\
\hline \multicolumn{13}{|l|}{ Education } \\
\hline Less than High School & 140 & 14.53 & 1.25 & 160 & 12.56 & 0.92 & 1 & - & - & 1 & - & - \\
\hline GED & 90 & 9.79 & 1.11 & 153 & 13.06 & 1.13 & $1.83 *$ & $1.16^{*}$ & $2.90 *$ & $1.76^{*}$ & $1.09 *$ & $2.85 *$ \\
\hline High School Diploma & 231 & 28.32 & 1.99 & 243 & 20.72 & 1.40 & 0.94 & 0.64 & 1.38 & 0.93 & 0.63 & 1.37 \\
\hline Any College & 392 & 46.57 & 2.11 & 627 & 53.02 & 1.75 & $1.42 *$ & $1.06 *$ & $1.90 *$ & $1.38^{*}$ & $1.02 *$ & $1.88^{*}$ \\
\hline \multicolumn{13}{|l|}{ Yearly Income } \\
\hline Below $\$ 25 \mathrm{k}$ & 419 & 45.64 & 2.23 & 552 & 43.34 & 1.79 & 0.94 & 0.75 & 1.17 & 0.97 & 0.77 & 1.22 \\
\hline Above $\$ 25 \mathrm{~K}$ & 382 & 47.23 & 2.22 & 568 & 50.45 & 1.98 & 1 & - & - & 1 & - & - \\
\hline
\end{tabular}


Table 2. Cont.

\begin{tabular}{|c|c|c|c|c|c|c|c|c|c|c|c|c|}
\hline Residence Type & & & & & & & & & & & & \\
\hline Missing & 250 & 19.90 & 1.58 & 291 & 16.80 & 1.06 & & & & & & \\
\hline Single family home & 392 & 51.18 & 1.96 & 595 & 55.65 & 1.77 & 1 & - & - & 1 & - & - \\
\hline $\begin{array}{l}\text { Multi-family home or apartment } \\
\text { complex }\end{array}$ & 191 & 25.10 & 1.63 & 259 & 23.76 & 1.53 & 0.83 & 0.65 & 1.06 & 0.89 & 0.70 & 1.13 \\
\hline Something else & 29 & 3.83 & 0.82 & 44 & 3.79 & 0.65 & 0.85 & 0.51 & 1.44 & 0.95 & 0.53 & 1.72 \\
\hline \multicolumn{13}{|l|}{ Own or Rent Home } \\
\hline Own & 302 & 37.36 & 2.18 & 375 & 33.75 & 1.50 & 1 & - & - & 1 & - & - \\
\hline Rent & 413 & 46.42 & 2.13 & 592 & 49.03 & 1.60 & 1.17 & 0.93 & 1.47 & 1.25 & 0.96 & 1.62 \\
\hline None of the Above & 136 & 14.88 & 1.33 & 215 & 16.60 & 1.19 & 0.29 & 0.93 & 1.80 & 1.39 & 0.97 & 2.00 \\
\hline \multicolumn{13}{|l|}{ Home Smoking Policy } \\
\hline Tobacco use prohibited at all times & 426 & 50.53 & 1.99 & 626 & 52.91 & 1.78 & 1 & - & - & 1 & - & - \\
\hline Any other policy & 432 & 48.84 & 1.99 & 562 & 46.91 & 1.77 & 0.92 & 0.73 & 1.17 & 1.05 & 0.82 & 1.36 \\
\hline \multicolumn{13}{|c|}{ Device Preference and ENDS Use Rationale } \\
\hline \multicolumn{13}{|l|}{ ENDS Device Most Frequently Used } \\
\hline Cartridge or disposable user & 543 & 62.79 & 1.89 & 628 & 54.70 & 1.81 & 1 & - & - & 1 & - & - \\
\hline Tank user & 285 & 32.40 & 1.70 & 529 & 42.89 & 1.74 & 1.49 & $1.21 *$ & $1.84 *$ & 1.47 * & 1.17 * & $1.85 *$ \\
\hline \multicolumn{13}{|l|}{$\begin{array}{l}\text { Use ENDS Because: they might be } \\
\text { less harmful to people around me } \\
\text { than cigarettes }\end{array}$} \\
\hline Yes & 614 & 72.31 & 1.75 & 1007 & 85.30 & 1.06 & $2.06^{*}$ & 1.57 * & $2.69 *$ & $2.10 *$ & $1.59 *$ & 2.76 * \\
\hline No & 245 & 27.15 & 1.73 & 179 & 14.55 & 1.06 & 1 & - & - & 1 & - & - \\
\hline \multicolumn{13}{|l|}{$\begin{array}{l}\text { Use/used e-cigarettes because: } \\
\text { They don't smell }\end{array}$} \\
\hline Yes & 538 & 63.85 & 1.74 & 901 & 76.95 & 1.48 & $1.90^{*}$ & $1.49 *$ & $2.41 *$ & $1.86 *$ & $1.43 *$ & $2.41 *$ \\
\hline No & 322 & 35.84 & 1.73 & 285 & 22.85 & 1.49 & 1 & - & - & 1 & - & - \\
\hline \multicolumn{13}{|l|}{$\begin{array}{l}\text { Use/used e-cigarettes because: } \\
\text { It comes/came in flavors I like/liked }\end{array}$} \\
\hline Yes & 520 & 58.11 & 1.79 & 905 & 74.75 & 1.43 & $1.99 *$ & $1.58 *$ & $2.49 *$ & $2.04 *$ & $1.62 *$ & $2.55 *$ \\
\hline No & 337 & 41.24 & 1.79 & 282 & 25.12 & 1.44 & 1 & - & - & 1 & - & - \\
\hline
\end{tabular}


Table 2. Cont

\begin{tabular}{|c|c|c|c|c|c|c|c|c|c|c|c|c|}
\hline \multicolumn{13}{|c|}{$\begin{array}{l}\text { Use/used e-cigarettes because: Using } \\
\text { them helps people to } \\
\text { quit smoking cigarettes }\end{array}$} \\
\hline Yes & 546 & 64.57 & 2.03 & 877 & 73.74 & 1.44 & $1.51 *$ & $1.20 *$ & $1.90 *$ & $1.52 *$ & $1.20 *$ & $1.92 *$ \\
\hline No & 315 & 35.26 & 2.02 & 309 & 26.03 & 1.44 & 1 & - & - & 1 & - & - \\
\hline \multicolumn{13}{|c|}{$\begin{array}{l}\text { Use/used e-cigarettes as an alternative } \\
\text { to quitting tobacco altogether }\end{array}$} \\
\hline Yes & 396 & 47.53 & 1.82 & 736 & 62.50 & 1.49 & $1.70 *$ & $1.37 *$ & $2.11 *$ & $1.71 *$ & $1.37 *$ & 2.13 * \\
\hline No & 464 & 52.14 & 1.82 & 448 & 37.18 & 1.48 & 1 & - & - & 1 & - & - \\
\hline \multicolumn{13}{|c|}{$\begin{array}{l}\text { Use/used e-cigarettes as a way of } \\
\text { cutting down on cigarette smoking }\end{array}$} \\
\hline Yes & 485 & 59.07 & 1.71 & 931 & 78.22 & 1.45 & $2.40 *$ & 1.90 * & $3.02 *$ & 2.38 * & $1.86 *$ & 3.05 * \\
\hline No & 376 & 40.89 & 1.71 & 257 & 21.70 & 1.46 & 1 & - & - & 1 & - & - \\
\hline \multicolumn{13}{|c|}{ Cessation and Dependence } \\
\hline \multicolumn{13}{|c|}{$\begin{array}{l}\text { Count of times over past } 12 \text { months, } \\
\text { stopped using tobacco for one day or } \\
\text { longer because trying to quit }\end{array}$} \\
\hline 0 times & 558 & 64.29 & 1.62 & 706 & 59.26 & 1.66 & 1 & - & - & 1 & - & - \\
\hline 1 time & 71 & 8.80 & 1.13 & 104 & 8.88 & 1.02 & 0.95 & 0.64 & 1.41 & 0.98 & 0.66 & 1.45 \\
\hline $2-3$ times & 153 & 17.72 & 1.41 & 237 & 20.04 & 1.28 & 1.20 & 0.93 & 1.55 & 1.23 & 0.95 & 1.61 \\
\hline $4+$ times & 80 & 9.19 & 0.98 & 142 & 11.82 & 0.99 & $1.46^{*}$ & $1.07 *$ & $2.00 *$ & $1.54 *$ & $1.12 *$ & $2.13 *$ \\
\hline \multicolumn{13}{|c|}{ Minutes after waking to first cigarette } \\
\hline less than $5 \mathrm{~min}$ & 192 & 23.32 & 1.67 & 273 & 22.75 & 1.17 & 1 & - & - & 1 & - & - \\
\hline 5-30 min & 303 & 33.32 & 1.61 & 443 & 36.97 & 1.61 & 1.15 & 0.91 & 1.46 & 1.13 & 0.88 & 1.46 \\
\hline $31-60 \mathrm{~min}$ & 165 & 19.71 & 1.48 & 220 & 18.94 & 1.30 & 1.00 & 0.72 & 1.39 & 1.00 & 0.71 & 1.40 \\
\hline $61+\min$ & 195 & 22.82 & 1.78 & 248 & 20.85 & 1.43 & 1.00 & 0.76 & 1.32 & 0.99 & 0.74 & 1.33 \\
\hline \multicolumn{13}{|c|}{$\begin{array}{l}\text { Minutes after waking to first } \\
\text { puff on ENDS }\end{array}$} \\
\hline Missing & 552 & 63.02 & 1.88 & 414 & 35.44 & 1.50 & & & & & & \\
\hline Less than 5 min & 71 & 8.41 & 1.28 & 138 & 11.26 & 0.92 & 1 & - & - & 1 & - & - \\
\hline 5-30 min & 74 & 8.52 & 1.00 & 196 & 16.73 & 1.34 & 1.39 & 0.86 & 2.23 & 1.47 & 0.82 & 2.63 \\
\hline $31-60 \mathrm{~min}$ & 53 & 5.55 & 0.65 & 148 & 12.45 & 0.95 & $1.83 *$ & $1.16 *$ & 2.88 * & $2.03 *$ & 1.17 * & 3.54 * \\
\hline $61+\min$ & 112 & 14.50 & 1.42 & 293 & 24.12 & 1.14 & 1.25 & 0.80 & 1.94 & 1.37 & 0.79 & 2.36 \\
\hline
\end{tabular}


Table 2. Cont.

\begin{tabular}{|c|c|c|c|c|c|c|c|c|c|c|c|c|}
\hline $\begin{array}{c}\text { Frequency of Daily Cigarette/ } \\
\text { ENDS Use }\end{array}$ & & & & & & & & & & & & \\
\hline Nondaily (Both Products) & 169 & 19.94 & 1.39 & 184 & 15.58 & 1.12 & 1 & - & - & 1 & - & - \\
\hline Nondaily Cigs, Daily ENDS & 40 & 4.97 & 0.90 & 158 & 13.14 & 0.98 & $3.00 *$ & $1.92 *$ & 4.67 * & 1.28 & 0.72 & 2.26 \\
\hline Daily (Both Products) & 41 & 5.49 & 0.93 & 142 & 12.39 & 0.98 & 2.67 * & 1.66 * & 4.28 * & 1.60 & 0.81 & 3.14 \\
\hline Daily Cigs, Nondaily ENDS & 612 & 69.60 & 1.71 & 705 & 58.89 & 1.72 & 1.02 & 0.81 & 1.28 & 0.63 & 0.39 & 1.04 \\
\hline \multicolumn{13}{|l|}{$\begin{array}{l}\text { Frequency of Daily ENDS Use, } \\
\text { Regardless of Cigarette Use }\end{array}$} \\
\hline Nondaily ENDS & 781 & 89.55 & 1.21 & 889 & 74.47 & 1.36 & 1 & - & - & 1 & - & - \\
\hline Daily ENDS & 81 & 10.45 & 1.21 & 300 & 25.53 & 1.36 & $2.79 *$ & $2.05 *$ & $3.81 *$ & $2.04 *$ & 1.39 * & 3.01 * \\
\hline \multicolumn{13}{|l|}{ Frequency of Daily Cigarette Use } \\
\hline Nondaily Cigarette User & 209 & 24.91 & 1.63 & 342 & 28.72 & 1.44 & 1 & - & - & 1 & - & - \\
\hline Daily Cigarette User & 653 & 75.09 & 1.63 & 847 & 71.28 & 1.44 & 0.79 & 0.64 & 0.97 & 0.65 & 0.43 & 1.00 \\
\hline
\end{tabular}




\section{Results}

The sociodemographic characteristics of the study population are given in Table 1. Of the 2051 dual users in the PATH Study, 1189 (58.0\%) users reported using ENDS in smoke-free places at least one time over the past 30 days. A majority of the population was male $(n=1035,53.4 \%)$, Caucasian $(n=$ $1644,81.92 \%)$, heterosexual $(n=1823,89.7 \%)$, and had completed at least some college $(n=1019,50.3 \%)$ Most of the study population consisted of nondaily ENDS users $(n=1670,80.9 \%)$ and daily cigarette smokers ( $n=1500,72.9 \%)$, respectively. A majority $(n=1621,79.8 \%)$ of the study population agreed that they used ENDS because of a belief that ENDS products may pose less risk to nearby nonusers compared to cigarettes, compared to 424 participants (19.9\%) that did not. More study participants used cartridge or disposable ENDS designs $(n=1171,58.1 \%)$ than tank systems and also agreed that they used ENDS because a variety of flavored products were available $(n=1425,67.7 \%)$. Agreement with prompts of ENDS use due to perceived lack of smell $(n=1439,71.4 \%)$, as a means to help quit smoking $(n=1423,69.9 \%)$, an alternative to quitting tobacco altogether $(n=1132,56.1 \%)$, and as a way to cut down on cigarette smoking $(n=1416,70.1 \%)$ were also prevalent.

The logistic regression model output is provided in Table 2. Gender and education were both significantly associated with increased odds of past 30-day ENDS use in smoke-free places. Men were significantly less likely than women to report using ENDS in smoke-free places, adjusting for all covariates (OR: 0.76, 95\% CI: 0.62, 0.93). Individuals whose highest level of education was a GED were significantly more likely to report ENDS use in smoke-free places (OR: 1.76, 95\% CI: 1.09, 2.85) than participants who reported less than high school diploma educational status, adjusting for all covariates. Similarly, individuals who reported any level of college completion were significantly more likely to report ENDS use in smoke-free places (OR: 1.38 95\% CI: 1.02,1.88) compared to respondents with less than high school diploma education status and adjusting for all covariates. Individual preference for tank ENDS designs was significantly associated with increased odds of ENDS use in smoke-free places compared to disposable or cartridge designs, after adjustment for all covariates (OR: 1.47, 95\% CI: 1.17, 1.85). Interestingly, frequency of cigarette use was not associated with change in odds of ENDS use in smoke-free places (OR: $0.65,95 \%$ CI: $0.43,1.00$ )

Among consumer characteristics, individuals who reported ENDS use because ENDS is "less harmful to nonusers than smoking" (OR: 2.10, 95\% CI: 1.59, 2.76), "ENDS do not smell" (OR: 1.86, 95\% CI: 1.43, 2.41), "[ENDS] are available in desirable flavors" (OR: 2.04, 95\% CI: 1.62, 2.55), "[ENDS] help smokers to quit cigarettes" (OR: 1.52, 95\% CI: 1.20, 1.92), believe that ENDS are an alternative to quitting tobacco (OR: 1.71, 95\% CI: 1.37, 2.13) or believe that ENDS help to cut down on cigarette smoking (OR: 2.38, 95\% CI: 1.86, 3.05) each were associated with increased likelihood of reported ENDS use in smoke-free places compared to individuals who did not share those beliefs, adjusting for all covariates. Dual users who self-reported ENDS use as a way of cutting down on cigarette use or as an alternative to quitting tobacco completely were both significantly more likely to report use of ENDS in smoke-free places compared to users who did not agree with those motivations for ENDS use. Cessation of tobacco use for one day or longer four or more times over the past year due to attempting to quit was also significantly associated with increased odds of ENDS use in smoke-free places (OR: 1.54, 95\% CI: 1.12, 2.13). Participants who were daily ENDS users were significantly more likely to report ENDS use in smoke-free places compared to nondaily ENDS users, adjusting for all covariates (OR: $2.04,95 \%$ CI: 1.39, 3.01).

\section{Discussion}

Although in-home use of ENDS products could not be directly assessed in this analysis, several characteristics of dual users were found to be significant predictors of ENDS use in other smoke-free places. Gender and education were both associated with significantly higher odds of reported ENDS use in smoke-free places. Men were less likely than women to report using ENDS in smoke-free places in this analysis. This finding may reflect greater awareness of ENDS use restrictions among men as men are more frequent users of ENDS than women. Participants who completed a GED or 
any college were more likely to report ENDS use in smoke-free spaces than individuals who did not complete high school; as was discussed above for gender, this finding may be due to the elevated use of ENDS in these populations. Individuals who agreed with beliefs that ENDS use is less harmful to passersby and reported using ENDS because of a belief that ENDS do not smell were both more likely to report using ENDS in smoke-free places than dual users who did not report either belief. This association is important for future policy actions, as various studies have suggested that ENDS emit detectable levels of nicotine and other pollutants into the immediate indoor environment, $[16,17]$ as discussed above. These quantifiable levels of nicotine in the indoor environment suggest that even though e-cigarettes may pose an attenuated risk of nicotine exposure compared to conventional tobacco cigarettes, nicotine-containing ENDS devices do introduce nicotine and other chemical constituents into indoor settings. Therefore, more extensive national counter-marketing and education campaigns should be undertaken in order to prevent the advancement of beliefs that ENDS confer no risk of passive exposure to nicotine and other chemicals in users and nonusers alike. Further, if there are no regulations on ENDS use in a given clean air setting, it is worth investigating why all ENDS users are not using their devices in clean air indoor environments. It is possible that the harm reductive beliefs described above are not as well-entrenched in the user base as the data might suggest. Despite this disparity, counter-marketing programs would likely benefit users and nonusers alike by attenuating the harm reduction beliefs that this analysis observed to be associated with increased odds of ENDS use in smoke-free places.

ENDS product design was found to be a significant predictor of past 30-day ENDS use in smoke-free places. ENDS users who preferred a tank design were observed to have higher odds of reporting ENDS use in smoke-free places compared to cartridge or disposable ENDS users, adjusting for all covariates. However, the relationship between ENDS product preference and ENDS use in smoke-free places is not well understood. Smoke-free legislation may also retroactively drive ENDS device preference in that some ENDS users may prefer e-products that are easier to conceal, further clouding the association between product selection and use of ENDS in smoke-free settings. For example, a dual user may be prompted to use a disposable or cartridge device, as these products may be more discrete than larger tank-based vaporizers. The observed trend between product preference and odds of ENDS use in smoke-free places corroborates the trend reported by Yingst et al. (2019), suggesting that a complex association may exist between product design and use of ENDS in smoke-free places [6]. Similarly, it is of note that the ENDS marketplace evolves rapidly, and replacement of tank systems by more popular JUUL products and other easily concealed ENDS products in recent years may influence current motivations for ENDS use in smoke-free places. The complex nature of this relation highlights the need for future research on the nature of the interaction between ENDS product design preference, attitudes associated with ENDS use, and reported vaping in smoke-free places.

Cessation beliefs were strongly associated with ENDS use in smoke-free places in this analysis. ENDS use as a smoking cessation tool is currently a topic of much debate in the field of tobacco control [46-49]. As described above, cessation perceptions and previous cessation attempts played a more prominent role than frequency of cigarette use in predicting ENDS use in smoke-free places. Further, there was no observed difference in odds of ENDS use in smoke-free places between frequencies of ENDS and conventional tobacco use. These significant associations suggest that holding beliefs that ENDS may serve as cessation aids may in turn contribute to increased use of e-products in places where conventional cigarettes are prohibited. Similarly, cessation beliefs were not mutually exclusive, meaning respondents may have had multiple rationales for continued ENDS use. Further, there was a clear positive trend in the relationship between past 12 month quit attempts lasting longer than one day and use of ENDS in smoke-free places. Dual users with the highest rate of quit attempts over the past year were significantly more likely to report ENDS use in smoke-free places than those who reported zero quit attempts, which provides more evidence in favor of a relationship between ENDS use as cessation devices and use of ENDS in smoke-free places. In general, holding pro-cessation beliefs towards ENDS products was associated with elevated odds of ENDS use in this analysis. Further, 
as described above, use of ENDS in enclosed spaces may result in deposition of nicotine onto surfaces in the indoor environment [15]. Nicotine present in the passive indoor environment is a source of nicotine exposure in adults, and third-hand smoke may potentially provide a mechanism by which ENDS use in indoor environments prolongs nicotine addiction in adults who wish to quit smoking. Much more research is required in order better describe this potential relationship. Data presented above therefore suggest that incorporating ENDS into existing smoke-free air legislation regulating public spaces may aid dual users attempting cessation in quitting tobacco and may also reduce the amount of passive nicotine that dual and nonusers may be exposed to in enclosed spaces.

There are several notable limitations in this study. One primary limitation is that as of this writing, there are no available state or county identifiers in the public Wave 2 PATH dataset, which precludes the investigation of differences between smoking patterns of users in smoke-free versus nonsmoke-free states or counties and the appraisal of compliance with smoke-free indoor air policies at a comprehensive level. The survey design also did not specify if the smoke-free place was a public or private place, which is an important point of clarity, as smoke-free indoor legislation does not cover privately-owned homes, meaning that ENDS use in these locations is regulated solely by the homeowner. Further, individuals who own their own homes may and should exert final authority over their behavior in their own home-therefore, policy recommendations drafted by findings of this analysis should only extend to shared public spaces. The cross-sectional nature of this study design is also a limitation, in that this analysis does not allow for changes in ENDS/cigarette dual use, cessation attempts, ENDS product preferences, or any of the other attitudinal or behavioral characteristics described above to be evaluated longitudinally. This study was performed as a cross-sectional rather than a longitudinal analysis because ENDS policy evolves slowly: The smoke-free air policy environment for 2013 (corresponding to Wave 1 of the PATH study) was remarkably similar to the national policy environment in 2014 [50]. Further, the American ENDS marketplace is constantly evolving; because this wave of the PATH survey was implemented between 2014 and 2015, investigation of the exponential increase in popularity and market share of newer ENDS products and designs such as JUUL (introduced and popularized in 2017) is not possible. Similarly, another limitation of this assessment is that the Wave 3 dataset has been available for several months as of this writing. Although the cross-sectional nature of this analysis precludes any evaluation of long-term shifts in product use or attitudinal changes in regard to public smoke-free indoor air policy compliance, the gradual nature of smoke-free air legislation implementation in the United States creates a legislative environment where policy differences between annual waves of Population Assessment on Tobacco or Health data are minimal. Therefore, this study provides a novel snapshot of ENDS use in public smoke-free places using a nationally-representative sample and in doing so provides important context and rationale for federal, state, and municipal smoke-free policy.

\section{Conclusions}

This study provides important context for ENDS use in public smoke-free places. Evidence from this study suggests that ENDS use as a cessation tool may place nonusers at greater possible risk for deleterious health effects if substitutive ENDS use occurs in a location that is currently regulated by clean air laws. Further, harm-reduction beliefs in ENDS users were associated with increased odds of ENDS use in public smoke-free places, suggesting that cessation and harm-reduction beliefs both play a role in determining an individual's likelihood to circumvent or disregard smoke-free air laws and policies. These findings both imply that a concerted education and counter-marketing campaign, combined with inclusion of ENDS into existing smoke-free policies, may provide critical protection for both ENDS/cigarette users and nonusers in public smoke-free places.

Author Contributions: Conceptualization, Z.R.D. and M.J.T.; methodology, G.G., M.L.G., R.J.O., B.W., and M.J.T., software and assessment, Z.R.D..; writing-original draft preparation, Z.R.D..; writing-review and editing, G.G., M.L.G., R.J.O., B.W., and M.J.T. All authors have read and agreed to the published version of the manuscript. 
Acknowledgments: This study was completed as part of a thesis dissertation.

Conflicts of Interest: The authors declare no conflict of interest.

\section{References}

1. Coleman, B.N.; Johnson, S.E.; Tessman, G.K.; Tworek, C.; Alexander, J.; Dickinson, D.M.; Rath, J.; Green, K.M. It's not smoke. It's not tar. It's not 4000 chemicals. Case closed: Exploring attitudes, beliefs, and perceived social norms of e-cigarette use among adult users. Drug Alcohol Depend. 2016, 159, 80-85. [CrossRef]

2. Berg, C.J.; Barr, D.B.; Stratton, E.; Escoffery, C.; Kegler, M. Attitudes toward E-Cigarettes, Reasons for Initiating E-Cigarette Use, and Changes in Smoking Behavior after Initiation: A Pilot Longitudinal Study of Regular Cigarette Smokers. Open J. Prev. Med. 2014, 4, 789-800. [CrossRef] [PubMed]

3. Brikmanis, K.; Petersen, A.; Doran, N. E-Cigarette Use, Perceptions, and Cigarette Smoking Intentions in a Community Sample of Young Adult Non-Daily Cigarette Smokers. Psychol. Addict. Behav. J. Soc. Psychol. Addict. Behav. 2017, 31, 336-342. [CrossRef] [PubMed]

4. Rass, O.; Pacek, L.R.; Johnson, P.S.; Johnson, M.W. Characterizing use patterns and perceptions of relative harm in dual users of electronic and tobacco cigarettes. Exp. Clin. Psychopharmacol. 2015, 23, 494-503. [CrossRef] [PubMed]

5. Shi, Y.; Cummins, S.E.; Zhu, S.-H. Use of electronic cigarettes in smoke-free environments. Tob. Control. 2017, 26, e19-e22. [CrossRef] [PubMed]

6. Yingst, J.M.; Lester, C.; Veldheer, S.; Allen, S.I.; Du, P.; Foulds, J. E-cigarette users commonly stealth vape in places where e-cigarette use is prohibited. Tobacco Control 2018. [CrossRef] [PubMed]

7. Yingst, J.M.; Veldheer, S.; Hammett, E.; Hrabovsky, S.; Foulds, J. Should electronic cigarette use be covered by clean indoor air laws? Tob. Control. 2017, 26, e16-e18. [CrossRef]

8. Lucherini, M.; Rooke, C.; Amos, A. E-cigarettes, vaping and performativity in the context of tobacco denormalisation. Sociol. Health Illn. 2018, 40, 1037-1052. [CrossRef]

9. Khachatoorian, C.; Jacob Iii, P.; Benowitz, N.L.; Talbot, P. Electronic cigarette chemicals transfer from a vape shop to a nearby business in a multiple-tenant retail building. Tob. Control. 2018. [CrossRef]

10. Cheng, T. Chemical evaluation of electronic cigarettes. Tob. Control. 2014, 23, ii11-ii17. [CrossRef]

11. Czogala, J.; Goniewicz, M.L.; Fidelus, B.; Zielinska-Danch, W.; Travers, M.J.; Sobczak, A. Secondhand exposure to vapors from electronic cigarettes. Nicotine Tob. Res. 2014, 16, 655-662. [CrossRef] [PubMed]

12. Goniewicz, M.L.; Knysak, J.; Gawron, M.; Kosmider, L.; Sobczak, A.; Kurek, J.; Prokopowicz, A.; Jablonska-Czapla, M.; Rosik-Dulewska, C.; Havel, C.; et al. Levels of selected carcinogens and toxicants in vapor from electronic cigarettes. Tob. Control. 2014, 23, 133-139. [CrossRef] [PubMed]

13. Schripp, T.; Markewitz, D.; Uhde, E.; Salthammer, T. Does e-cigarette consumption cause passive vaping? Indoor Air 2013, 23, 25-31. [CrossRef] [PubMed]

14. Volesky, K.D.; Maki, A.; Scherf, C.; Watson, L.; Van Ryswyk, K.; Fraser, B.; Weichenthal, S.A.; Cassol, E.; Villeneuve, P.J. The influence of three e-cigarette models on indoor fine and ultrafine particulate matter concentrations under real-world conditions. Environ. Pollut. 2018. [CrossRef]

15. Jacob, P.; Benowitz, N.L.; Destaillats, H.; Gundel, L.; Hang, B.; Martins-Green, M.; Matt, G.E.; Quintana, P.J.E.; Samet, J.M.; Schick, S.F.; et al. Thirdhand smoke: New evidence, challenges, and future directions. Chem. Res. Toxicol. 2017, 30, 270-294. [CrossRef]

16. Goniewicz, M.L.; Lee, L. Electronic cigarettes are a source of thirdhand exposure to nicotine. Nicotine Tob. Res. 2015, 17, 256-258. [CrossRef]

17. Bush, D.; Goniewicz, M.L. A pilot study on nicotine residues in houses of electronic cigarette users, tobacco smokers, and non-users of nicotine-containing products. Int. J. Drug Policy 2015, 26, 609-611. [CrossRef]

18. Yeramaneni, S.; Dietrich, K.N.; Yolton, K.; Parsons, P.J.; Aldous, K.M.; Haynes, E.N. Secondhand tobacco smoke exposure and neuromotor function in rural children. J. Pediatrics 2015, 167, 253-259. [CrossRef]

19. Yolton, K.; Dietrich, K.; Auinger, P.; Lanphear, B.P.; Hornung, R. Exposure to environmental tobacco smoke and cognitive abilities among U.S. children and adolescents. Environ. Health Perspect 2005, 113, 98-103. [CrossRef]

20. Anderko, L.; Braun, J.; Auinger, P. Contribution of tobacco smoke exposure to learning disabilities. J. Obstet. Gynecol. Neonatal Nurs. 2010, 39, 111-117. [CrossRef] 
21. Hecht, S.S.C.; Chi-hong, B.; Ohmori, T.; Hoffmann, D. Comparative carcinogenicity in F344 rats of the tobacco-specific nitrosamines, N'-Nitrosonornicotine and 4-(N-Methyl-N-nitrosamino)-1-(3-pyridyl)1-butanone. Cancer Res. 1980, 40, 298-302.

22. Balbo, S.; James-Yi, S.; Johnson, C.S.; O'Sullivan, M.G.; Stepanov, I.; Wang, M.; Bandyopadhyay, D.; Kassie, F.; Carmella, S.; Upadhyaya, P.; et al. (S)-N'-Nitrosonornicotine, a constituent of smokeless tobacco, is a powerful oral cavity carcinogen in rats. Carcinogenesis 2013, 34, 2178-2183. [CrossRef]

23. Hecht, S.S. It is time to regulate carcinogenic tobacco-specific nitrosamines in cigarette tobacco. Cancer Prev. Res. (Philadelphia Pa.) 2014, 7, 639-647. [CrossRef]

24. Terzano, C.; Di Stefano, F.; Conti, V.G.E.; Petroianni, A. Air pollution ultrafine particles: Toxicity beyond the lung. Eur. Rev. Med. Pharmacol. Sci. 2010, 14, 809-821.

25. Dunbar, Z.R.; Das, A.; O'Connor, R.J.; Goniewicz, M.L.; Wei, B.; Travers, M.J. Brief report: Lead levels in selected electronic cigarettes from Canada and the United States. Int. J. Environ. Res. Public Health 2018, 15, 154. [CrossRef]

26. Hess, C.A.; Olmedo, P.; Navas-Acien, A.; Goessler, W.; Cohen, J.E.; Rule, A.M. E-cigarettes as a source of toxic and potentially carcinogenic metals. Environ. Res. 2017, 152, 221-225. [CrossRef]

27. Williams, M.; Villarreal, A.; Bozhilov, K.; Lin, S.; Talbot, P. Metal and silicate particles including nanoparticles are present in electronic cigarette cartomizer fluid and aerosol. PLoS ONE 2013, 8, e57987. [CrossRef]

28. King, A.C.; Smith, L.J.; McNamara, P.J.; Matthews, A.K.; Fridberg, D.J. Passive exposure to electronic cigarette (e-cigarette) use increases desire for combustible and e-cigarettes in young adult smokers. Tob. Control. 2015, 24, 501-504. [CrossRef]

29. US Centers for Disease Control and Prevention. E-Cigarette Use Among Youth and Young Adults: A Report of the Surgeon General; US Centers for Disease Control and Prevention: Atlanta, GA, USA, 2016.

30. Chen, C.; Zhuang, Y.-L.; Zhu, S.-H. E-Cigarette design preference and smoking cessation: A U.S. population study. Am. J. Prev. Med. 2016, 51, 356-363. [CrossRef]

31. Glasser, A.M.; Collins, L.; Pearson, J.L.; Abudayyeh, H.; Niaura, R.S.; Abrams, D.B.; Villanti, A.C. Overview of electronic nicotine delivery systems: A systematic review. Am. J. Prev. Med. 2017, 52, e33-e66. [CrossRef]

32. Adkison, S.E.; O'Connor, R.J.; Bansal-Travers, M.; Hyland, A.; Borland, R.; Yong, H.-H.; Cummings, K.M.; McNeill, A.; Thrasher, J.F.; Hammond, D.; et al. Electronic nicotine delivery systems: International tobacco control four-country survey. Am. J. Prev. Med. 2013, 44, 207-215. [CrossRef] [PubMed]

33. Rutten, L.J.F.; Blake, K.D.; Agunwamba, A.A.; Grana, R.A.; Wilson, P.M.; Ebbert, J.O.; Okamoto, J.; Leischow, S.J. Use of e-cigarettes among current smokers: Associations among reasons for use, quit intentions, and current tobacco use. Nicotine Tob. Res. 2015, 17, 1228-1234. [CrossRef] [PubMed]

34. Lechner, W.V.; Tackett, A.P.; Grant, D.M.; Tahirkheli, N.N.; Driskill, L.M.; Wagener, T.L. Effects of duration of electronic cigarette use. Nicotine Tob. Res. 2015, 17, 180-185. [CrossRef] [PubMed]

35. Berg, C.J. Preferred flavors and reasons for e-cigarette use and discontinued use among never, current, and former smokers. Int. J. Public Health 2016, 61, 225-236. [CrossRef]

36. Farsalinos, K.E.; Romagna, G.; Tsiapras, D.; Kyrzopoulos, S.; Voudris, V. Characteristics, perceived side effects and benefits of electronic cigarette use: A worldwide survey of more than 19,000 consumers. Int. J. Environ. Res. Public Health 2014, 11, 4356-4373. [CrossRef]

37. Family Smoking Prevention And Tobacco Control And Federal Retirement Reform. In 111-31, Congress, t., Ed. 2009; Vol. H.R. 1256, pp 123 Stat. 1776-1123 Stat. 1858. Available online: https://www.congress.gov/bill/ 111th-congress/house-bill/1256 (accessed on 3 February 2020).

38. US Food and Drug Administration. Deeming Tobacco Products To Be Subject to the Federal Food, Drug, and Cosmetic Act, as Amended by the Family Smoking Prevention and Tobacco Control Act; Restrictions on the Sale and Distribution of Tobacco Products and Required Warning Statements for Tobacco Products; Administration, U.S.F.a.D., Ed.; US Food and Drug Administration: Washington, DC, USA, 2016.

39. Centers for Disease Control and Prevention. The Call to Action on E-Cigarette Use Among Youth and Young Adults: A Report of the Surgeon General; U.S. Department of Health and Human Services, C.f.D.C.a.P.; National Center for Chronic Disease Prevention and Health Promotion, Office on Smoking and Health, Eds.; Centers for Disease Control and Prevention: Atlanta, GA, USA, 2016; pp. 235-251.

40. Foundation, A.N.R. United States 100\% Smokefree Air Laws. Available online: https://no-smoke.org/wpcontent/uploads/pdf/100Map.pdf (accessed on 9 July 2018). 
41. Foundation, A.N.R. Summary of $100 \%$ Smokefree State Laws and Population Protected by $100 \%$ U.S. Smokefree Laws. Available online: https://no-smoke.org/wp-content/uploads/pdf/SummaryUSPopList.pdf (accessed on 2 February 2019).

42. Hyland, A.; Ambrose, B.K.; Conway, K.P.; Borek, N.; Lambert, E.; Carusi, C.; Taylor, K.; Crosse, S.; Fong, G.T.; Cummings, K.M.; et al. Design and methods of the Population Assessment of Tobacco and Health (PATH) Study. Tob. Control. 2017, 26, 371-378. [CrossRef]

43. Pericot-Valverde, I.; Gaalema, D.E.; Priest, J.S.; Higgins, S.T. E-cigarette awareness, perceived harmfulness, and ever use among U.S. adults. Prev. Med. 2017, 104, 92-99. [CrossRef]

44. Link, A.R.; Cawkwell, P.B.; Shelley, D.R.; Sherman, S.E. An Exploration of Online Behaviors and Social Media Use Among Hookah and Electronic-Cigarette Users. Addict. Behav. Rep. 2015, 2, 37-40. [CrossRef]

45. Apelberg, B.J.; Corey, C.G.; Hoffman, A.C.; Schroeder, M.J.; Husten, C.G.; Caraballo, R.S.; Backinger, C.L. Symptoms of tobacco dependence among middle and high school tobacco users: Results from the 2012 National Youth Tobacco Survey. Am. J. Prev. Med. 2014, 47, S4-S14. [CrossRef]

46. Brown, J.; Beard, E.; Kotz, D.; Michie, S.; West, R. Real-world effectiveness of e-cigarettes when used to aid smoking cessation: A cross-sectional population study. Addiction 2014, 109, 1531-1540. [CrossRef]

47. Pokhrel, P.; Herzog, T.A. Reasons for quitting cigarette smoking and electronic cigarette use for cessation help. Psychol. Addict. Behav. J. Soc. Psychol. Addict. Behav. 2015, 29, 114-121. [CrossRef] [PubMed]

48. Tan, A.S.L.; Bigman, C.A. E-Cigarette Awareness and Perceived Harmfulness: Prevalence and Associations with Smoking Cessation Outcomes. Am. J. Prev. Med. 2014, 47, 141-149. [CrossRef] [PubMed]

49. McRobbie, H.; Bullen, C.; Hartmann-Boyce, J.; Hajek, P. Electronic cigarettes for smoking cessation and reduction. Cochrane Database Syst. Rev. 2014. [CrossRef]

50. Holmes, C.B.; King, B.A.; Babb, S.D. Stuck in Neutral: Stalled Progress in Statewide Comprehensive Smoke-Free Laws and Cigarette Excise Taxes, United States, 2000-2014. Prev. Chronic Dis. 2016, 13, E80. [CrossRef]

(C) 2020 by the authors. Licensee MDPI, Basel, Switzerland. This article is an open access article distributed under the terms and conditions of the Creative Commons Attribution (CC BY) license (http://creativecommons.org/licenses/by/4.0/). 